\title{
Integration of clinicopathological and mutational data offers insight into lung cancer with tumor spread through air spaces
}

\author{
Yu Tian ${ }^{1 \#}$, Jing Feng ${ }^{2 \#}$, Long Jiang ${ }^{1} \wedge$, Junwei Ning $^{1}$, Zenan Gu ${ }^{1}$, Jia Huang ${ }^{1}$, Qingquan Luo ${ }^{1}$ \\ ${ }^{1}$ Shanghai Lung Cancer Center, Shanghai Chest Hospital, Shanghai Jiao Tong University, Shanghai, China; ${ }^{2}$ Statistical Center, Shanghai Chest \\ Hospital, Shanghai Jiao Tong University, Shanghai, China \\ Contributions: (I) Conception and design: Q Luo, J Huang, Y Tian; (II) Administrative support: J Huang, Q Luo; (III) Provision of study materials \\ or patients: J Huang, J Feng; (IV) Collection and assembly of data: J Feng, L Jiang; (V) Data analysis and interpretation: Y Tian, J Feng; (VI) \\ Manuscript writing: All authors; (VII) Final approval of manuscript: All authors. \\ \#These authors contributed equally to this work. \\ Correspondence to: Qingquan Luo; Jia Huang. Shanghai Lung Cancer Center, Shanghai Chest Hospital, Shanghai Jiao Tong University, Shanghai \\ 200030, China. Email: luoqingquan@hotmail.com; huangjiadragon@126.com.
}

\begin{abstract}
Background: Tumor spread through air spaces (STAS) was defined as a unique tumor invasion pattern in adenocarcinoma (ADC) by The World Health Organization Classification of Lung Tumors in 2015. Since then, STAS had been shown to be associated with local recurrence and poor survival results, as the typical signature and potential mechanisms of STAS remained unclear. Our objectives were to comprehensively demonstrate the clinicopathological and genetic signatures in STAS-positive lung cancer patients.

Methods: The clinicopathological and gene alteration characteristics of 878 STAS-positive lung cancer patients were presented. Associations between parameters were evaluated using the Chi-square test, Fisher's exact test, and logistic regression. The capture-based targeted next generation sequencing (NGS) with a platform of 68 lung cancer-related genes was conducted in 139 cases, and the mutational spectrum was summarized.

Results: STAS was identified in 391 female and 481 male patients, of which ADC accounted for the majority of cases $(92.6 \%)$. The concomitant solid or micropapillary subtype was observed in $92.12 \%$ patients with ADC. Poorly differentiated histological subtypes were more frequent and negatively correlated with tumor size in smaller tumor cases $(\mathrm{P}=0.036$, Pearson's $\mathrm{R}=-0.075)$. Furthermore, in the subgroup of nodules within $3 \mathrm{~cm}$, the distribution of the solid and micropapillary subtypes were significantly frequent in lymph node-positive patients $(\mathrm{P}<0.001)$. Tumor protein p53 (TP53) alterations were more frequent in smoking patients $(27.6 \%, \mathrm{P}=0.007)$, human epidermal growth factor receptor 2 (HER2) alterations were more common in female $(10.8 \%, \mathrm{P}=0.025)$, while Kirsten rat sarcoma viral oncogene (KRAS) $(20.3 \%, \mathrm{P}=0.024)$ and TP53 (45.9\%, P=0.003) were more prevalent in males.

Conclusions: Poorly differentiated histological subtypes likely played a crucial role in promoting the invasiveness of STAS, especially in small tumor-size cases. Epidermal growth factor receptor (EGFR), TP53, KARS, anaplastic lymphoma kinase (ALK), and ROS proto-oncogene 1 (ROS1) were the five most frequent alterations in STAS-positive ADC.
\end{abstract}

Keywords: Lung cancer; adenocarcinoma (ADC); spread through air space; pathological; genetic

Submitted Apr 16, 2021. Accepted for publication Jun 15, 2021.

doi: $10.21037 /$ atm-21-2256

View this article at: https://dx.doi.org/10.21037/atm-21-2256

^ ORCID: Yu Tian, 0000-0002-5252-9374; Long Jiang, 0000-0002-6860-755X. 


\section{Introduction}

Lung cancer is the leading cause of cancer-related death, and has a complicated prognosis. With the advancement of imaging, pathological, and molecular detection techniques for lung cancer, the identification, diagnosis, and staging of patients is becoming more comprehensive and individualized (1,2). Early and precise discrimination of tumor features have become the key to achieving optimal treatment modality and improved prognosis.

Tumor invasion is one of the decisive factors of tumor treatment and prognosis. Compared with other organ cancers, the tumor invasion patterns in lung adenocarcinoma (ADC) are diverse. The common patterns of pulmonary carcinoma invasion are via vascular, lymphatic, or transcoelomic spread. The concept of spread through air spaces (STAS) was included as a unique pattern of invasion in lung ADC in the World Health Organization (WHO) classification in 2015 (3). In other histological types (i.e., besides ADC), STAS has also been described (4).

Recent studies regarding STAS have focused on its value in therapeutic decision-making and prognostic assessment. The presence of STAS was validated as a risk factor of pulmonary recurrence in patients undergoing limited reception (5). STAS was also associated with significantly decreased recurrence free survival and overall survival (6). Several studies attempted to identify the predictive factors of STAS, however their conclusions remained controversial $(7,8)$.

Therefore, this study aimed to demonstrate the clinicopathological and genetic signatures of STAS in lung cancer and offer a scale for more comprehensive understanding of the disease. Furthermore, we also expected to explore and analyze the critical features influencing the invasiveness of lung cancer STAS. We presented the following article in accordance with the STROBE reporting checklist (available at https://dx.doi.org/10.21037/atm-21-2256).

\section{Methods}

\section{Study cohort and data collection}

Between February 2015 and February 2020, 939 patients were pathologically validated as STAS-positive. Of these, 10 cases of bilateral surgeries and 28 cases of biopsy or palliative surgeries were excluded. Of the remaining 901 patients, 878 underwent lymph node systematic dissection or sampling and were finally enrolled. Next generation sequencing (NGS) data was obtained in 139 cases. The study was conducted in accordance with the Declaration of Helsinki (as revised in 2013). The study was approved by institutional ethics committee of Shanghai Chest Hospital [No.: KS(Y) 21128] and informed consent was taken from all the patients.

Data on clinicopathologic variables, including age, gender, smoking status, tumor location, pathologic tumornode-metastasis (TNM) situation, visceral pleural invasion, lymphovascular invasion, histological classification, and subtype, were obtained by reviewing the patients' medical records. Staging was on the basis of the eighth edition of the American Joint Committee on Cancer Staging Manual.

\section{Capture-based targeted DNA sequencing}

Tested samples were identified and derived from surgical resected tissue by the pathologist before pathological examination, which followed the principle of anonymity and voluntariness. DNA was sheared using Covaris M220 (Covaris, MA, USA), fragments of size 200-400 base pairs (bp) were selected by bead (Agencourt AMPure XP Kit, Beckman Coulter, Brea, CA, USA). Qubit 2.0 fluorometer with the dsDNA high-sensitivity assay kit (Life Technologies, Carlsbad, CA, USA) was used to measure the DNA concentration and genomic DNA quality. Indexed samples were sequenced on Nextseq500 sequencer (Illumina, Inc., Madison, WI, USA) with pair-end reads. We assessed the genomic profiles using Lung Core panel from Burning Rock Biotech (Guangzhou, China), which consists of the whole exons of 68 lung cancer-related genes spaning $345 \mathrm{~kb}$ of the human genome.

\section{Statistical analysis}

Connections between clinicopathologic and genetic parameters were analyzed using the Chi-square test or Fisher's exact test for categorical parameters. A P value less than 0.05 was considered to indicate a significant difference. All statistical analyses were performed through IBM SPSS Statistics v.23 (IBM, Armonk, NY, USA) and R software (version 4.0.1, the R Foundation for Statistical Computing, Vienna, Austria).

\section{Results}

\section{Patient clinicopathological features of STAS}

The clinicopathological characteristics of STAS-positive lung cancer patients who underwent surgery were summarized in 
Sex, n (\%)

Smoking status, $\mathrm{n}(\%)$

Never

Former or current

$187(21.3)$

Histological type, n (\%)

Adenocarcinoma

$813(92.6)$

Squamous cell carcinoma

$25(2.8)$

Others

Tumor size, $\mathrm{cm}$

$2.80 \pm 1.64$

Visceral pleural invasion, $\mathrm{n}(\%)$

Present

323 (36.8)

Absent

555 (63.2)

Lymphovascular invasion, $\mathrm{n}$ (\%)

Present

261 (29.7)

Absent

Pathologic N stage, n (\%)

NO

N1

N2

Pathologic T stage, n (\%)

T1

T2

T3

T4

$8^{\text {th }}$ TNM stage, n (\%)

।

II

III

IV

Disseminated nodule, $\mathrm{n}(\%)$

Present

51 (5.8)

Absent

827 (94.2)

Tumor location, n (\%)

Left upper

$211(24.0)$

Left lower

$176(20.0)$

Right upper

227 (25.9)

Right middle

65 (7.4)

Right lower

199 (22.7)

Year

2015-2017
Table 1 . The mean age of the patients was $60.75 \pm 10.14$ years, and 487 of the 878 patients $(55.5 \%)$ were males. The proportion of patients with smoking history was $21.3 \%$ $(\mathrm{n}=187)$. The most frequent histological type was ADC $(\mathrm{n}=813,92.6 \%)$, followed by squamous cell carcinoma $(\mathrm{n}=25,2.8 \%)$. The median pathological tumor size was $2.80 \pm 1.64 \mathrm{~cm}$. Of the 878 patients, 548 cases $(62.4 \%)$ were pathologically diagnosed as N0, 87 cases $(9.9 \%)$ as $\mathrm{N} 1$, and 243 cases $(27.7 \%)$ as N2; 484 cases were classified as pathologic stage I, 123 were stage II, 261 were stage III, and 11 were stage IV. As for other invasive patterns, visceral pleural invasion was observed in 323 cases (36.8\%), lymphovascular invasion was observed in 261 cases (29.7\%), and pulmonary disseminated nodule was observed in 51 cases $(5.8 \%)$.

\section{STAS and histological subtype}

Histologically, in 813 ADC patients, 336 cases (41.4\%) were classified as acinar predominant type, 168 (20.7\%) as papillary predominant, $136(16.7 \%)$ as micropapillary predominant, $118(14.5 \%)$ as solid predominant, $29(3.6 \%)$ as cribriform predominant, five $(0.6 \%)$ as lepidic predominant, and $21(2.6 \%)$ as others variants (including mucinous, colloid, fatal and enteric subtypes) (Figure 1A).

As the solid and micropapillary subtypes were validated as poor prognostic factors in lung ADC, the presence of poorly differentiated subtypes was particularly concerning. The concomitant solid or micropapillary subtype was observed in $89.0 \%$ (299 cases) of acinar predominant, $97.0 \%$ (163 cases) of papillary predominant, $72.4 \%$ (21 cases) of cribriform predominant, $60 \%$ (three cases) of lepidic predominant, and $42.9 \%$ (nine cases) of other variant predominant cases. The pure micropapillary and solid types were observed in 13 and 25 cases, respectively (Figure 1B).

\section{Association between pathological subtypes and other invasive patterns}

According to recent studies, STAS was significantly associated with tumor size. Tumor invasion, including STAS, was more likely to be observed as the burden of tumor volume increased. Compared with cases of larger tumor size, the poorly differentiated histological subtypes were more frequent in smaller tumor-size patients $(\mathrm{P}=0.036)$. Also, tumor size was negatively correlated with differentiated grade in STAS-positive ADC patients (Pearson's $\mathrm{R}=-0.075$ ) (Figure 2). Furthermore, when 

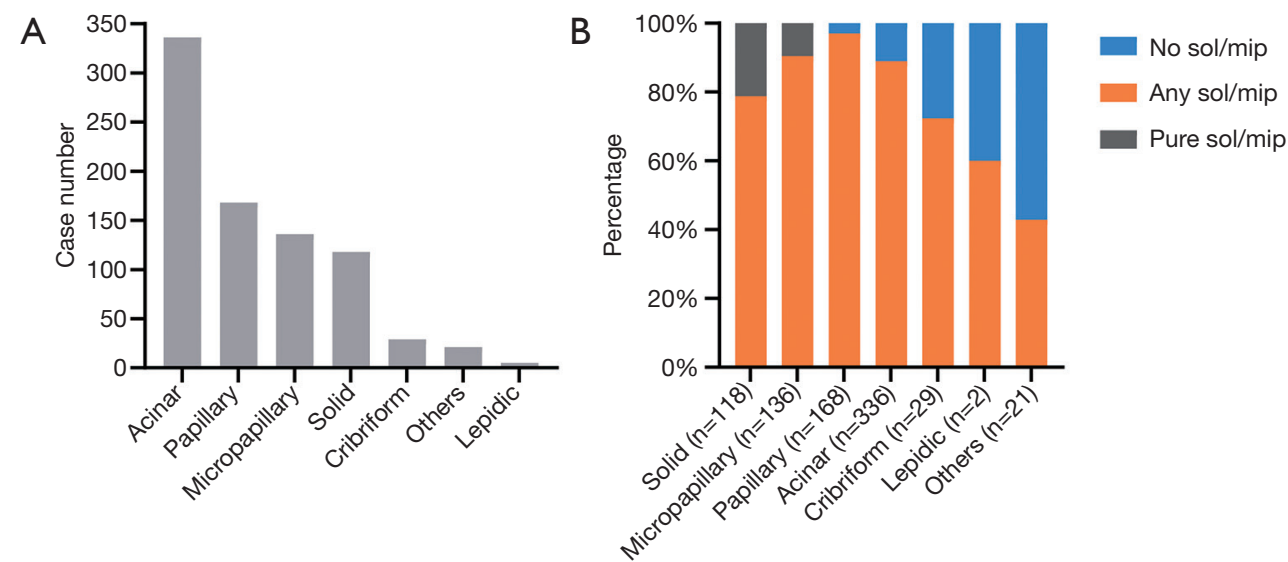

Figure 1 Distribution of predominant subtypes and poorly differentiated components in STAS-positive adenocarcinoma. (A) The number of cases of different predominant histological subtypes in 813 adenocarcinoma patients. (B) The proportion of cases with concomitant solid or micropapillary components among different histological types.

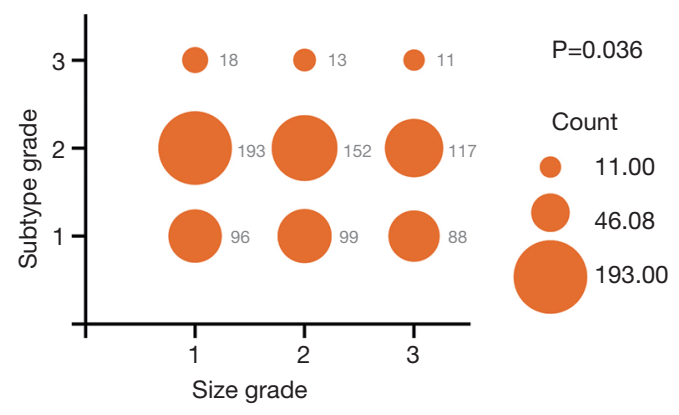

Figure 2 The correlation between tumor size and differentiation grade of histological type in STAS-positive adenocarcinoma patients. Bubble sizes were proportional to case numbers; analysis was performed using the Mantel-Haenszel hi-square test (size grade 1: nodule $\leq 2 \mathrm{~cm}$, size grade $2: 2 \mathrm{~cm}<$ nodule $\leq 3 \mathrm{~cm}$, size grade 3 : nodule $>3 \mathrm{~cm}$; subtype grade 1 : tumor with pure solid or micropapillary or cribriform subtypes, subtype grade 2: tumor with concomitant solid or micropapillary or cribriform subtypes, subtype grade 3: tumor without any solid or micropapillary or cribriform subtypes). STAS, spread through air spaces.

the tumor size was restricted within $3 \mathrm{~cm}$, histological distribution were significantly different in lymph nodepositive patients $(\mathrm{P}<0.001)$, the solid and micropapillary subtypes were more frequent (Table 2), and the presence of these two components was a risk factor of lymph node metastasis in STAS $(\mathrm{OR}=3.63,95 \% \mathrm{CI}, 1.26-10.40$, $\mathrm{P}=0.02$ ) (Table 3).

\section{Gene mutational signature in STAS}

In total, 139 surgically resected STAS-positive ADC tissue samples were tested with NGS panel of 68 cancer-related genes. The main driver genes and specific oncogenes were included in this analysis. The comprehensive genetic spectra of patients with STAS showed that all of them harbored genetic abnormalities (Figure 3). EGFR mutation was detected in $51.8 \%$ of the 139 patients. Second to EGFR, 35\% of patients harbored TP53, and the proportion of patients with KRAS, ALK, and ROS1 were 14\%, 12\%, and $8 \%$, respectively. The different distribution of genetic alterations based on clinicopathological characteristics was further explored. TP53 alterations were more frequent in patients with smoking history $(27.6 \%, \mathrm{P}=0.007)$. HER2 alterations were more common in females $(10.8 \%$, $\mathrm{P}=0.025)$, while KRAS (20.3\%, $\mathrm{P}=0.024)$ and TP53 (45.9\%, $\mathrm{P}=0.003$ ) were more prevalent in males.

\section{Discussion}

The novelty of this study is reflected in its summary of the comprehensive features of STAS, including clinicopathological information and gene sequencing data, using a large sample size of STAS patients. The majority of STAS-positive cases were ADC, which was characterized by a broad range of histologic subtypes and driver or targetable mutations. The differentiation grade subtype was found to be negatively correlated with tumor size in STASpositive ADC. Lymph node metastasis was also found to 
Table 2 Histological subtype distribution among different lymph-node stages

\begin{tabular}{|c|c|c|c|c|c|}
\hline Variable & \multicolumn{3}{|c|}{ Pathological lymph node stage } & Total & $P$ value \\
\hline Papillary & 89 & 7 & 24 & 120 & $<0.001$ \\
\hline Acinar & 201 & 11 & 44 & 256 & \\
\hline Micropapillary & 56 & 7 & 28 & 91 & \\
\hline Cribriform & 18 & 1 & 4 & 23 & \\
\hline Total & 404 & 39 & 128 & & \\
\hline
\end{tabular}

Table 3 Logistic regression analysis of factors associated with lymph node metastasis in STAS

\begin{tabular}{|c|c|c|c|c|c|c|}
\hline Factor & \multicolumn{3}{|c|}{ Univariable analysis } & \multicolumn{3}{|c|}{ Multivariable analysis } \\
\hline Age & 0.99 & $0.97-1.01$ & 0.35 & 0.99 & $0.97-1.01$ & 0.33 \\
\hline Male (vs. female) & 1.05 & $0.73-1.51$ & 0.78 & 1.01 & $0.67-1.51$ & 0.97 \\
\hline Smoking (vs. never) & 1.06 & $0.67-1.68$ & 0.79 & 1.15 & $0.69-1.91$ & 0.59 \\
\hline Pleural invasion & 2.40 & $1.65-3.49$ & $<0.001$ & 2.09 & $1.42-3.08$ & $<0.001$ \\
\hline Presence of SOL/MIP & 3.63 & $1.26-10.40$ & 0.02 & 3.26 & $1.10-9.68$ & 0.03 \\
\hline
\end{tabular}

STAS, spread through air spaces; SOL, solid; MIP, micropapillary.

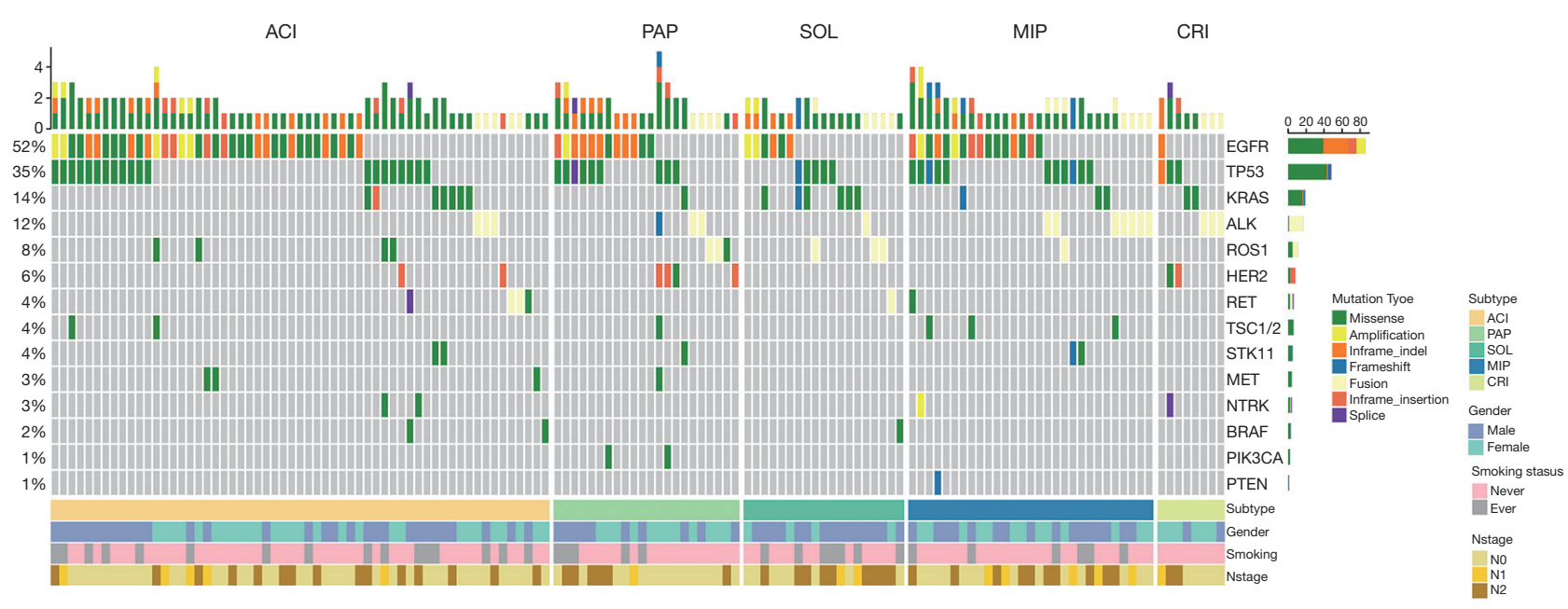

Figure 3 Mutational spectrum of the 139 STAS-positive adenocarcinoma patients grouped according to predominant histological subtypes. Smoking status, gender and lymph node stage were also annotated at the bottom of the oncoprint. Each column represented a patient and each row represented a gene. The column on the left represented the mutation rate of each gene. The column on the right represented mutated genes. The top plot represented the overall number of mutations that a patient carried. The different colors denoted different types of mutations. STAS, spread through air spaces. 
be associated with poorly differentiated subtypes. These results indicated that the histological subtypes seemed to take a larger facilitative effect on STAS presence and other invasive processes when the burden of the tumor itself was relatively small. Meanwhile, some frequent mutant genes, such as EGFR, TP53, KRAS, and ALK, were displayed.

STAS, as a unique invasion pattern of lung cancer, has been referred to frequently in recent years. The WHO 2015 classification proposed its definition as one or more pathologic micropapillary clusters, solid nests, or single cells beyond the edge of the tumor into air spaces in the surrounding lung parenchyma. However, the identification and further classification of STAS remained undefined. The presence of STAS was notably controversial when the phenomenon was close to the tumor edge or was presented as several isolated tumor cells (9). The most common subtype grouping method of STAS was based on morphological features including single cell, small clusters, and tumor cell nests, which was similar to the WHO classification. The grade of STAS was also grouped according to distance from primary lesions (10) or quantity of spread cells or clusters (4).

Recently, an increasing number of studies have focused on STAS due to its significant prognostic value. Dai et al. found that stage I ADC patients with STAS had a remarkable decrease in recurrence free survival (RFS) and overall survival (OS) (11). Also, patients with stage IA ADC shared a similar prognosis with stage IB, which suggested the STAS could be a factor leading to the up-staging of lung cancer, like visceral pleural invasion. Patients with STAS undergoing limited resection were found to have a higher rate of local recurrence and related-death in previous studies (6), indicating that STAS could be the explanation for the poorer prognosis of some patients receiving limited resection with a relatively small nodule and suggesting that surgical range should be expanded in STAS-positive patients accepting limited resection. Furthermore, the STAS was shown to be a predictor of occult lymph node metastasis (12). Another study demonstrated that the impact of STAS on survival was particularly evident in papillary predominant and micropapillary predominant $\mathrm{ADC}$ (10). In the present study, poorly differentiated ADC subtypes, such as solid and micropapillary components, were more likely the driving factors in tumor invasion and possible poor prognosis.

Since the important prognostic role of STAS in earlystage lung cancer, early identification and even prediction are critical in guiding surgical methods and improve prognosis. An important finding reported by Eguchi et al. (6) was that pathologists were able to recognize STAS on intraoperative frozen sections with high sensitivity and specificity (71\% and $92 \%$, respectively). However, in research by Walts et $a l$., frozen sections seemed to be unreliable for evaluating the presence of STAS (13). Meanwhile, recent studies have shown that imaging information, such as tumor diameter larger than $2 \mathrm{~cm}$, solid component of nodules, and higher F-fluorodeoxyglucose (FDG) uptake of primary lesion, could help identify the presence of STAS $(5,14)$. Kim et al. reported that the percentage of solid component of the lesion on CT (but not tumor diameter) was an independent predictor of STAS (15). Besides the preoperative prediction, optimal adjuvant therapy should be considered for patient with STASpositive ADC. Further studies based on multidisciplinary collaboration are needed to improve the preoperative and intraoperative identification of STAS.

In terms of molecular alteration, the conclusions of current studies are conflicting. Several studies have found that STAS was negatively associated with EGFR mutation and positively correlated with BRAF and KRAS mutations, while others have reported no association to EGFR (14) or KRAS (16) status. STAS has also been found to be more frequent in tumors with ROS1 and ALK rearrangements (15). In the present research, EGFR, TP53, KARS, ALK, and ROS1 were the five most frequent alterations in STAS-positive ADC, which indicated that some of these high frequent mutations might play a promoting role in STAS development. The mutation rate of EGFR was comparable with the Asian population (17). The frequency of KARS, ALK, and ROS1 partly supported the findings of previous studies. As for TP53, the mutation of this conventional anti-oncogene may promote the presence of STAS via unknow mechanism. More genomic profiling is expected to determine the typical molecular alterations and potential driver mutation of STAS.

Some limitations of this study included the fact that a control group was missing, as well as deficiency in the study design and data sources. Another limitation was the lack of survival results due to the majority of the patients having visited hospital after 2019 as well as the insufficient followup duration. Retrospective examination of pathological sections was not conducted, which led to reevaluation and detailed classification of STAS. Moreover, the NGS was not performed in all STAS-positive patients; larger sequencing data sizes could be more convincing and have a greater potential to discover significant results.

In conclusion, the present study comprehensively demonstrated the clinicopathological and genetic signatures in STAS-positive lung cancer patients. The 
poorly differentiated histological subtypes were shown to play a crucial role in promoting the invasiveness of STAS, especially in small tumor-size cases. As far as we know, this was by far the largest cohort of STAS-positive lung cancer patients. The NGS results and mutational spectrum were first used to profile the comprehensive molecular signature. The result of this research was expected to provide direction for further investigation on the dominating factors and molecular mechanisms of STAS presence.

\section{Acknowledgments}

Funding: This study was supported by grants from the National Foundation for Natural Scientific Research of China (grant No. 8197101926).

\section{Footnote}

Reporting Checklist: The authors have completed the STROBE reporting checklist. Available at https://dx.doi. org/10.21037/atm-21-2256

Data Sharing Statement: Available at https://dx.doi. org/10.21037/atm-21-2256

Conflicts of Interest: All authors have completed the ICMJE uniform disclosure form (available at Available at https:// dx.doi.org/10.21037/atm-21-2256). The authors have no conflicts of interest to declare.

Ethical Statement: The authors are accountable for all aspects of the work in ensuring that questions related to the accuracy or integrity of any part of the work are appropriately investigated and resolved. The study was conducted in accordance with the Declaration of Helsinki (as revised in 2013). The study was approved by institutional ethics committee of Shanghai Chest Hospital (No.: KS(Y) 21128) and informed consent was taken from all the patients.

Open Access Statement: This is an Open Access article distributed in accordance with the Creative Commons Attribution-NonCommercial-NoDerivs 4.0 International License (CC BY-NC-ND 4.0), which permits the noncommercial replication and distribution of the article with the strict proviso that no changes or edits are made and the original work is properly cited (including links to both the formal publication through the relevant DOI and the license).
See: https://creativecommons.org/licenses/by-nc-nd/4.0/.

\section{References}

1. de Koning HJ, van der Aalst CM, de Jong PA, et al. Reduced Lung-Cancer Mortality with Volume CT Screening in a Randomized Trial. N Engl J Med 2020;382:503-13.

2. Imyanitov EN, Iyevleva AG, Levchenko EV. Molecular testing and targeted therapy for non-small cell lung cancer: Current status and perspectives. Crit Rev Oncol Hematol 2021;157:103194.

3. Travis WD, Brambilla E, Nicholson AG, et al. The 2015 World Health Organization Classification of Lung Tumors: Impact of Genetic, Clinical and Radiologic Advances Since the 2004 Classification. J Thorac Oncol 2015;10:1243-60.

4. Uruga H, Fujii T, Fujimori S, et al. Semiquantitative Assessment of Tumor Spread through Air Spaces (STAS) in Early-Stage Lung Adenocarcinomas. J Thorac Oncol 2017;12:1046-51.

5. Shiono S, Yanagawa N. Spread through air spaces is a predictive factor of recurrence and a prognostic factor in stage I lung adenocarcinoma. Interact Cardiovasc Thorac Surg 2016;23:567-72.

6. Eguchi T, Kameda K, Lu S, et al. Lobectomy Is Associated with Better Outcomes than Sublobar Resection in Spread through Air Spaces (STAS)-Positive T1 Lung Adenocarcinoma: A Propensity Score-Matched Analysis. J Thorac Oncol 2019;14:87-98.

7. Jiang C, Luo Y, Yuan J, et al. CT-based radiomics and machine learning to predict spread through air space in lung adenocarcinoma. Eur Radiol 2020;30:4050-7.

8. Li C, Jiang C, Gong J, et al. A CT-based logistic regression model to predict spread through air space in lung adenocarcinoma. Quant Imaging Med Surg 2020;10:1984-93.

9. Kadota K, Nitadori JI, Sima CS, et al. Tumor Spread through Air Spaces is an Important Pattern of Invasion and Impacts the Frequency and Location of Recurrences after Limited Resection for Small Stage I Lung Adenocarcinomas. J Thorac Oncol 2015;10:806-14.

10. Warth A, Muley T, Kossakowski CA, et al. Prognostic Impact of Intra-alveolar Tumor Spread in Pulmonary Adenocarcinoma. Am J Surg Pathol 2015;39:793-801.

11. Dai C, Xie H, Su H, et al. Tumor Spread through Air Spaces Affects the Recurrence and Overall Survival in Patients with Lung Adenocarcinoma $>2$ to $3 \mathrm{~cm}$. J Thorac Oncol 2017;12:1052-60. 
12. Vaghjiani RG, Takahashi Y, Eguchi T, et al. Tumor Spread 2014;9:154-62. Through Air Spaces Is a Predictor of Occult Lymph Node Metastasis in Clinical Stage IA Lung Adenocarcinoma. J Thorac Oncol 2020;15:792-802.

13. Walts AE, Marchevsky AM. Current Evidence Does Not Warrant Frozen Section Evaluation for the Presence of Tumor Spread Through Alveolar Spaces. Arch Pathol Lab Med 2018;142:59-63.

14. Toyokawa G, Yamada Y, Tagawa T, et al. Significance of Spread Through Air Spaces in Resected Pathological Stage I Lung Adenocarcinoma. Ann Thorac Surg 2018;105:1655-63.

15. Kim SK, Kim TJ, Chung MJ, et al. Lung Adenocarcinoma: CT Features Associated with Spread through Air Spaces. Radiology 2018;289:831-40.

16. Lee JS, Kim EK, Kim M, et al. Genetic and clinicopathologic characteristics of lung adenocarcinoma with tumor spread through air spaces. Lung Cancer 2018;123:121-6.

17. Shi Y, Au JS, Thongprasert S, et al. A prospective, molecular epidemiology study of EGFR mutations in Asian patients with advanced non-small-cell lung cancer of adenocarcinoma histology (PIONEER). J Thorac Oncol

(English Language Editor: A. Kassem)

Cite this article as: Tian Y, Feng J, Jiang L, Ning J, Gu Z, Huang J, Luo Q. Integration of clinicopathological and mutational data offers insight into lung cancer with tumor spread through air spaces. Ann Transl Med 2021;9(12):985. doi: 10.21037/atm-21-2256 